\title{
Representações de gestantes sobre o uso de plantas medicinais
}

\author{
RANGEL, M. ${ }^{1^{*}}$; BRAGANÇA, F.C.R. ${ }^{2}$ \\ Universidade do Estado do Rio de Janeiro, Faculdade de Ciências Médicas, Rio de Janeiro, RJ. Rua São \\ Francisco Xavier, 524, Maracanã, CEP 20550-013. E-mail da Instituição:posgraduacaoemcienciasmedicas@ \\ yahoo.com.br; * mrangel@abel.org.br. Universidade Federal Fluminense, Faculdade de Educação. Campus do \\ Gragoatá, Centro, Niterói, RJ - CEP24230-030.e-mail da Instituição:poseduc@vm.uff.br; ${ }^{2}$ Universidade Federal \\ Fluminense, Faculdade de Medicina. Rua Marquês do Paraná, 303, Centro, Niterói, RJ - CEP 24230-030. e-mail da \\ Instituição:mgm@vm.uff.br.
}

\begin{abstract}
RESUMO: Esta pesquisa insere-se no campo da etnofarmacologia e utiliza, como fonte de dados, informações de gestantes usuárias, ou não, de fitoterápicos. O objetivo foi o de analisar representações formadas sobre o uso de plantas medicinais por gestantes em tratamento ambulatorial. Na perspectiva metodológica, assinala-se a natureza qualitativa do estudo e o emprego da análise de conteúdo, na perspectiva bardiniana. O levantamento de dados foi feito com um grupo de gestantes que usava e um grupo que não usava fitoterápicos. As análises permitiram identificar as categorias da concepção e dos motivos do uso, ou não, das plantas, verificando-se, com o grupo de usuárias, que o seu conhecimento vem sendo transmitido de geração a geração, principalmente através da informação verbal. Pôde-se, então, observar que esse conhecimento, embora aceito, pode levar ao uso inadequado ou ineficaz. No grupo que não usava fitoterápicos, pôde-se perceber desconhecimento da fitoterapia, ou a compreensão das plantas como perigosas, prejudiciais ao feto. Nas conclusões, verificaram-se dificuldades de identificação e administração segura das plantas e um certo grau de mistificação, além do uso de substâncias ineficazes e potencialmente tóxicas, destacando-se, então, o valor do diálogo entre médico e pacientes, com atenção ao possível uso medicinal de plantas.
\end{abstract}

Palavras-chave: gravidez, etnofarmacologia, representações sociais, plantas medicinais

\begin{abstract}
Representations of pregnant women on the use of medicinal plants. The present ethnopharmacological study evaluated information by pregnant women using phytotherapy or not. Its aim was to analyze representations on the use of medicinal plants by pregnant women under outpatient care. As regards methodology, the qualitative nature of the study and the content analysis underlie the bardinian approach. Data were collected from two groups: phytotherapyuser and non-user pregnant women. Categories related to the concept and the reason of plant use or non-use were identified. In the users group, knowledge has been transmitted over generations, mainly through verbal information. Although such knowledge has been accepted, it can lead to an unsuitable or inefficient use. The group of non-users was unaware of phytotherapy and considered plants dangerous and harmful to the fetus. In conclusion, there were difficulties regarding plant identification and safe administration, as well as certain mysticism, in addition to the use of inefficient and potentially toxic substances. Thus, the dialogue between doctors and patients focused on the possible medicinal use of plants must be emphasized.
\end{abstract}

Key words: pregnancy, ethnopharmacology, social representations, medicinal plants

Recebido para publicação em 07/01/2008

Aceito para publicação em 15/05/2008

Rev. Bras. PI. Med., Botucatu, v.11, n.1, p.100-109, 2009. 


\section{INTRODUÇÃO}

Desde épocas remotas, as sociedades humanas acumulaminformações e experiência sobre o ambiente que as cerca, para com ele interagir e prover suas necessidades de sobrevivência. Nesses acervos, inscreve-se o conhecimento relativo ao mundo vegetal com o qual essas sociedades estão em contato. No desenvolvimento de diversas civilizações, prevalece uma estreita relação entre a mulher e as plantas.

A história revela que um número crescente de espécies utilizadas na medicina tradicional vem sendo incorporado às prescrições médicas para o tratamento de agravos à saúde. Estima-se que o número de espécies vegetais superiores descrito possa chegar a 750.000 e que 119 substâncias químicas extraídas de plantas indicadas para fins medicinais são obtidas de aproximadamente 90 espécies. Desses compostos químicos, cerca de $75 \%$ derivam de plantas usadas na medicina tradicional (Dutau, 2004; Brasil, 2006).

A descoberta de medicamentos a partir de vegetais representa uma das formas de evolução da ciência. O desenvolvimento da etnofarmacologia, da farmacologia experimental e da química de produtos naturais levou ao isolamento, à determinação de estruturas (De Smet, 2002) e identificação do mecanismo de ação de princípios ativos e substâncias como: atropina e hioscina (antiespasmódicos), colchicina (anti-reumático), digital (cardiotônico), ópio (modelo de potentes analgésicos), metformina (antidiabético), pilocarpina (antiglaucoma), salicilatos (analgésicos e antiinflamatórios) e reserpina (hipotensor e sedativo). Segundo David \& David (2006), um indício do renascimento de fármacos derivados de fontes vegetais é o progresso de pesquisas clínicas no campo dos agentes anticancerígenos (taxol, podofilotoxina e camptotecinas) e dos antimaláricos (artemisina).

A pesquisa sobre determinada planta, em algumas ocasiões, é realizada com uma finalidade inicial e, no decorrer dos ensaios farmacológicos e clínicos, são descobertas propriedades mais importantes. É interessante o exemplo da vinca-rosa, Catharanthus roseus $\mathrm{G}$. Don, originária de Madagascar e popularmente conhecida no Brasil como "maria-sem-vergonha". Essa espécie é fonte de pelo menos 60 alcalóides (Guerra \& Nodari, 2001). O estudo de sua ação hipoglicemiante permitiu determinar propriedades leucopenizantes e o isolamento de duas substâncias de grande valor: vincristina e vimblastina, empregadas no tratamento da leucemia.

O National Cancer Chemotherapy Program vem testando cerca de 120.000 extratos vegetais com possíveis atividades anticancerígenas, de 35.000 diferentes espécies obtidas de fontes espalhadas por todo o mundo. Vale ressaltar que muitas dessas plantas foram escolhidas exclusivamente a partir da evidência popular do seu valor medicinal. Segundo estimativa da OMS, que relacionou 2.000 espécies vegetais, $80 \%$ da população dos países em desenvolvimento dependem diretamente de plantas medicinais para seu tratamento (Organização Panamericana de Saúde, 2002; Laloo et al., 2006).

Mesmo com a globalização da indústria química e a utilização de medicamentos sintéticos, os produtos derivados de plantas medicinais ainda detêm uma parcela do mercado mundial, 14 bilhões de umtotal estimado de 280 bilhões de dólares, cerca de $5 \%$ do mercado mundial de produtos farmacêuticos (Carvalho et al., 2007).

Na China, a interação dos conhecimentos tradicionais com a visão científico-tecnológica contemporânea tem-se mostrado altamente positiva. No Brasil, com cerca de $30 \%$ das florestas tropicais do planeta, admite-se que existam entre 55 mil e 80 mil espécies vegetais somente na Amazônia brasileira e que menos de $2 \%$ delas foram estudadas pelos cientistas (Castro \& Ferreira, 2001).

Considerando-se as especificações adotadas para a enumeração da carga microbiana presente e pesquisa de microrganismos específicos, verificouse que mais de $90 \%$ das espécies vegetais estão em desacordo com um ou mais parâmetros microbiológicos. Esses dados sugerem que drogas vegetais podem ser consideradas produtos de alto risco (Fugh-Berman, 2000), sendo necessário respeitar as medidas de controle higiênico-sanitário definidas pela Agência Nacional de Vigilância Sanitária (ANVISA), para garantir sua qualidade e segurança, coleta, armazenamento e manipulação, até o produto final (Bugno et al., 2005).

Efeitos colaterais não estão restritos à farmacologia de medicamentos sintéticos. Produtos fitoterápicos têm sido apresentados na mídia como recursos terapêuticos alternativos, isentos de contraindicações ou toxicidade. No entanto, conhecimentos científicos e mesmo empíricos questionam (quando não negam) essas informações (Choudhuri \& Valerio, 2005). A representação de que "o natural não faz mal" precisa ser analisada e essa análise interessa, de modo especial, ao uso de plantas na gestação (Fugh-Berman et al., 2005).

\section{O uso de plantas na gestação}

O uso medicinal de plantas é comum na gestação (Lacroix et al., 2000). Esse período, especialmente sensível na vida e no comportamento da mulher, abrange dimensões socioculturais, históricas e afetivas, que acentuam a sua sensibilidade. Complementarmente, observa-se que circulam, no meio social, concepções, práticas e 
modelos explicativos populares da gestação que, em muitos aspectos, diferenciam-se do modelo biomédico, podendo, inclusive, conflitar com princípios científicos de proteção da mãe e do feto (Calvasina et al., 2007). A gestação é considerada momento especial, tanto pela ciência quanto pela cultura. Assim, a mulher segue determinadas condutas baseadas em saberes provenientes das culturas familiar e biomédica (Baião \& Deslandes, 2007).

A saúde da mulher na gestação tem suscitado atenções especiais, tanto no campo da ciência, como no da cultura popular. Nesse período, a mulher é particularmente sensível à influência de orientações e "conselhos" de familiares e amigos sobre "remédios caseiros", considerados úteis à sua saúde (Fonseca et al., 2002).

Os vegetais costumam ser complexos do ponto de vista fitoquímico, podendo conter substâncias tóxicas, além dos princípios biologicamente ativos.

A produção do conhecimento científico sobre plantas medicinais evidencia contribuições e possíveis riscos do uso de espécies da flora brasileira (Mendonça Filho \& Menezes, 2003). Por outro lado, é importante qualificar o conhecimento das camadas populares sobre seus aspectos benéficos à saúde $\mathrm{e}$, nesse sentido, ressalta-se a contribuição do estudo de representações sobre o uso das plantas, para que se possa confrontá-las com conceitos e parâmetros científicos, no interesse das necessárias orientações e esclarecimento a respeito (Souza, 2007). Esse mesmo tipo de interesse justificou o objetivo desta pesquisa, sustentado por seu referencial teórico.

\section{Objetivo, referencial teórico e perspectiva metodológica}

Levando em conta que interessam à etnofarmacologia as informações coletadas dentro de umdeterminado grupo de contexto semelhante (Etkin \& Elisabetsky, 2005), esta pesquisa teve como objetivo analisar representações formadas sobre o uso de plantas medicinais por gestantes em tratamento ambulatorial, reunindo informações que possam auxiliar a compreender, estudar e discutir esse uso.

Conhecer o perfil dos medicamentos usados na gravidez pode ajudar a planejar programas de esclarecimento para pacientes e de educação continuada para profissionais de saúde. Em um estudo retrospectivo sobre o padrão do uso de medicamentos durante a gestação, realizado em hospital-escola brasileiro, Fonseca e colaboradores (2002) observaram que $11,2 \%$ foram utilizados por automedicação ou por indicação leiga. Problemas dessa natureza realçam as possíveis contribuições desta pesquisa e seus aportes teóricos e conceituais.

O referencial teórico incorporou estudos sobre etnofarmacologia e representação social. O estudo farmacológico de plantas pode ser dividido em farmacologia de produtos naturais e etnofarmacologia. Enquanto na primeira abordagem os vegetais são estudados pela farmacologia clássica, na segunda levam-se emconsideração as informações coletadas dentro de um determinado grupo culturalmente definido (grupo de contexto semelhante), usuário dos produtos (fármacos) a serem estudados (Aschwanden, 2001).

A etnofarmacologia, segundo Mendonça Filho \& Menezes (2003), tem se mostrado uma boa fonte de novas substâncias e drogas, quando comparada a outros métodos de descoberta (aleatório, quimiossistemático e filogenético). É importante estabelecer essa diferenciação, tendo em vista que os medicamentos tradicionais já não são produtos naturais e simartefatos humanos, isto é, uma certa espécie, preparada e ingerida de maneira específica, que possui uma determinada ação terapêutica (Albuquerque \& Hanazaki, 2006).

A partir dessa concepção, as informações etnofarmacológicas são usadas como base para investigações, que objetivamo estudo das espécies enquanto fármacos potenciais (Di Stasi, 2002). Uma breve reflexão acerca das possíveis contribuições das pesquisas que investigam conceitos e significados atribuídos aos objetos do cotidiano dos grupos sociais, entre as quais se situa a pesquisa de representações, pode demonstrar a sua importância às questões da saúde.

$\mathrm{Na}$ perspectiva moscoviciana, as representações sociais são compreendidas como uma modalidade específica de conhecimento, que tem por função a compreensão de fatos e fenômenos, através de seus conceitos, formados nas comunicações e interações dos indivíduos na vida e experiência cotidianas. As representações são constituídas de conceitos e imagens circulantes nos grupos sociais, sobre objetos de seu uso e interesse (Arruda, 2002). Observa-se a possibilidade de que as representações reflitam aspectos da realidade dos fatos, uma vez que sua formação associa-se às experiências cotidianas dos indivíduos, repercutindo emsuas percepções, conceitos e na forma como se comunicame interagem (Moscovici, 2005).

Sublinha-se, então, o entendimento de que as representações apresentam-se como um conhecimento prático, que se forma no contexto de vida dos sujeitos, com possibilidade de influir em suas crenças e expectativas. As representações se expressam através de conceitos e imagens, que revelam o modo de compreensão dos fatos, divulgado e consolidado na comunicação e interação dos sujeitos nos grupos sociais dos quais participam.

É interessante reler Spink (1995, p.119), quando assinala uma "nova perspectiva", que "amplia o conhecimento, objeto de estudo, para além das 
fronteiras da ciência, passando a abarcar, também, o conhecimento de senso comum".

Jodelet (apud Guareschi, 1995) focaliza, no conceito de representação social, "uma forma de conhecimento socialmente elaborada e partilhada, tendo uma visão prática e concorrendo para a construção de uma realidade comum a um conjunto social".

Constata-se, portanto, a fecundidade das representações sociais como marco analítico em diversas áreas de conhecimento e, em particular, no campo da saúde e da doença (Jodelet, 2001; Minayo, 2006). Uma das tarefas da pesquisa pode ser, então, a de indicar de que modo essas representações estão enraizadas na realidade, no cotidiano social, ao mesmo tempo em que também contribuem para construí-la, na medida emque influem nas convicções e ações dos sujeitos. Autores como Sá $(1996,1998)$, Santiago (2000), Schopenhauer (2001), Goffman (2002), Guareschi (2000) afinam-se emseus aportes teóricos, oferecendo subsídios expressivos à pesquisa de representações, com fundamentos no campo moscoviciano.

Finalmente, em seu estudo epistemológico sobre o fenômeno das representações, Moscovici $(1978,1988,2001,2005)$ demonstra que algumas ciências, como a sociologia, a antropologia e a psicologia, desde cedo se preocuparam em compreender o pensamento e a conduta dos indivíduos, com atenção aos conceitos que influem sobre ambos. Contudo, o autor considera que, apesar das contribuições dos estudos realizados por essas ciências, eles apresentam resultados fragmentados. Essa fragmentação pode estar associada ao fato das pesquisas permaneceram fechadas em enfoques específicos, ora privilegiando o caráter coletivo das representações, ora destacando os conceitos individuais como substratos psíquicos e fatores de interiorização e subjetividade desses conceitos, preocupando-se pouco em considerar a comunicação que permite aos indivíduos interagireme convergirem, de sorte que as percepções e conceitos individuais podem se tornar sociais e vice-versa.

Quanto à perspectiva metodológica, é interessante, preliminarmente, observar, no campo de pesquisas de representações, uma tendência atual de incremento ao uso de análises quantitativas, notando-se, inclusive, a freqüente recorrência ao uso de programas, a exemplo do software EVOC. O tratamento quali-quantitativo está sendo crescentemente, considerado (Rangel, 2004). Contudo, nesta pesquisa de representações de gestantes sobre o uso medicinal de plantas, as análises orientaram-se pelo tratamento qualitativo de dados, obtidos através da compreensão das falas dos sujeitos, com atenção às dimensões da representação social e aos núcleos de sentido, identificados através da constância de idéias, que constituirão as categorias resultantes da análise. A base do encaminhamento metodológico encontra-se na análise de conteúdo, de acordo comBardin (1986).

Ainda, na opção metodológica, considerouse Minayo (2006), quando observa que, entre as principais características das pesquisas de natureza qualitativa, destaca-se a preocupação com o entendimento do significado que os sujeitos atribuem ao objeto da investigação. Esse entendimento foi o principal escopo dessa investigação.

No tratamento qualitativo, não se utilizou a perspectiva de população e amostra, mas sim de grupos de sujeitos. Embora não se tenha aplicado, propriamente, a técnica de saturação, procurou-se observar, nas falas das gestantes, o ponto em que as idéias começaram a se repetir, notando-se, nessa repetição, não só o aspecto de sua redundância, como também evidências de sua constância e semelhanças.

Ressalta-se também, no levantamento de dados, o princípio de que, nas representações individuais, encontram-se expressões da formação social do pensamento (Vala, 1986). Comesse princípio metodológico da pesquisa de representações, reafirmase a relevância, para o presente estudo, das idéias que se expressaramnas afirmações de cada um dos sujeitos (entendendo essas afirmações como resultantes de uma formação social de idéias), assim como a relevância das idéias que foram mais comuns, na expressão coletiva de cada um dos grupos de gestantes.

A escolha de gestantes atendeu, também, a um dos critérios da pesquisa de representações, referido ao estudo com grupos que apresentam características comuns e elementos homogêneos, considerando-se, ainda, a facilidade de contato do pesquisador, como médico que as assistia. Observase, também, que a OMS tem priorizado o grupo materno-infantil para receber os cuidados de saúde, especialmente por ser mais vulnerável aos riscos de adoecer e morrer (Andrade et al., 2004) e por representar a maioria da população em países subdesenvolvidos (Cecatti, 2005).

Assim, o grupo de sujeitos da pesquisa constituiu-se de 139 (cento e trinta e nove) gestantes, assistidas no Hospital Universitário Antônio Pedro e na Policlínica Comunitária Carlos Antonio da Silva, em Niterói, Estado do Rio de Janeiro, Brasil, no ano de 2003. Formaram-se, a partir de adesão espontânea ao convite para participação na pesquisa, dois grupos, sendo um com79 (setenta e nove) gestantes usuárias e outro com60 (sessenta) não usuárias de vegetais, para uso medicinal. Ainvestigação com esses dois grupos de sujeitos possibilitou reunir informações em circunstâncias diversas, o que interessa especialmente ao estudo das representações sociais. 
Levando-se, novamente, em conta que, na perspectiva moscoviciana, a representação de um sujeito revela uma formação social de idéias, considera-se o número de 139 (cento e trinta e nove) sujeitos satisfatório à proposta deste estudo, no sentido, não de esgotar ou generalizar resultados, mas sim de obter dados que possam trazer contribuições à discussão do objeto da representação: o uso de plantas medicinais por gestantes.

Oinstrumento de pesquisa foi composto por questões abertas, que permitiram a livre expressão dos sujeitos na resposta à indagação sobre se utilizavam, ou não, plantas para uso medicinale por quê.

O levantamento de dados foi feito através da técnica de entrevista não-diretiva. Foi feita uma aplicação experimental da técnica. Não se tratou de um projeto piloto, mas apenas de um teste, feito com 10 (dez) gestantes, 5 (cinco) usuárias e 5 (cinco) não usuárias, que atenderam espontaneamente ao convite para participarem. O objetivo do teste foi apenas o de verificar a compreensão e possíveis dificuldades dos sujeitos em responderem à indagação feita. Nessa aplicação, verificou-se que não houve dificuldade de compreensão e resposta, mantendo-se, então, a formulação original da questão: Você utiliza, ou não, plantas como remédio? Em caso afirmativo, quais? Por quê? Em caso negativo, por quê?

Quanto aos aspectos éticos, o estudo seguiu os termos da Resolução n 196/96 do Conselho Nacional de Ética em Pesquisa e foi aprovado pelo Comitê de Ética e Pesquisa da Universidade Federal Fluminense, sobre a documentação referente ao protocolo de pesquisa. Por ocasião da coleta de dados, solicitou-se às gestantes entrevistadas que assinassemo termo de consentimento informado (livre e esclarecido), enfatizando-se a liberdade para participarem do estudo, a ausência de qualquer ônus e a garantia de anonimato.

Com esse encaminhamento metodológico, chegam-se aos dados e análises, iniciando-se por observar e tecer alguns comentários sobre os vegetais citados pelas gestantes usuárias e as espécies mais utilizadas.

\section{Vegetais citados pelas gestantes e espécies mais utilizadas}

Ao todo, 39 vegetais foram citados 133 vezes e as espécies mais utilizadas foram: erva-cidreira $(79,5 \%)$, boldo $(41 \%)$, erva-doce $(28 \%)$ e camomila (28\%). O grupo de gestantes usuárias dispõe de um elenco de plantas que, na sua maioria, não recebeu confirmação científica de suas ações no tratamento de agravos à saúde. Essas plantas são utilizadas como parte integrante dos cuidados domésticos e como estratégia empregada pelas famílias para evitar doenças (Gibson, 2001). Merece destaque o exemplo do Cymbopogon citratus, Staph., planta da família das gramíneas, originária da Ásia, cujos nomes vulgares foram os mais citados neste trabalho: erva cidreira, capim limão, capim santo, capim cidreira, capim-cidró, citronela, capim de cheiro e capim cidrão. Seu óleo essencial (citral) temsido relatado como indutor de hipotensão arterial e reações de sensibilização em humanos, quando utilizado isoladamente. O hidrolato desse vegetal provocou, em alguns casos de superdosagem: hipocinesia, ataxia, bradipnéia, perda de postura, sedação e diarréia (Teske \& Trentini, 2001).

O boldo (Peumus boldus Molina) possui propriedades colagogas, além de ser estimulante hepático e leve demulcente urinário. Seu óleo volátil é considerado um dos mais tóxicos, contendo terpenos irritantes. Doses excessivas podem provocar alterações renais, não havendo segurança comprovada para a sua administração no período gestacional (Newall et al., 2002).

As ações farmacológicas da erva-doce (Pinpinella anisum L.), considerada abortiva e galactagoga, devem-se, em grande parte, à presença do anetol, que está estruturalmente relacionado com as catecolaminas adrenalina, noradrenalina e dopamina. Os dímeros de anetol assemelham-se bastante aos agentes estrogênicos: estilbeno e estilboestrol. O óleo é carminativo e expectorante, com possíveis efeitos simpaticomiméticos. As dermatites de contato com a erva-doce e com seu óleo foram atribuídas ao anetol. Aingestão de $5 \mathrm{~mL}$ do óleo pode provocar náuseas, vômitos, convulsões e edema pulmonar (Newall et al., 2002; De Smet, 2002).

As espécies de Matricaria recutita L. e Chamaemelum nobile (L.) All. possuem atividades farmacológicas semelhantes, como atividades antiinflamatórias e antiespasmódicas. São comuns relatos de reações alérgicas à camomila, embora na maioria dos casos a espécie da planta não tenha sido especificada. Os indivíduos que tenham apresentado hipersensibilidade a qualquer membro da família Asteraceae/Compositae (por exemplo, à ambrósia) devem evitar a camomila, tida popularmente como desreguladora do ciclo menstrual. Seus extratos são uterotônicos e seu uso excessivo no período de gravidez e amamentação deve ser contra-indicado (Newall, et al., 2002).

As fibras de Plantago ovata são utilizadas no tratamento da constipação intestinal. O grande risco associado ao seu uso está na ingestão insuficiente de líquidos, podendo levar à obstrução esofágica, especialmente nos quadros de motilidade gastrintestinal prejudicada (Turolla \& Nascimento, 2006). Segundo estes autores, outra planta utilizada tradicionalmente é o Panax ginseng, usada pela 
Medicina Tradicional Chinesa na forma de chás e outras formas. A administração continuada foi associada à ocorrência de sangramento vaginal, mastalgia e alterações do estado mental e, menos freqüentemente: hipertensão, insônia, erupções cutâneas e diarréia matinal. Há trabalhos que descrevem a fototoxicidade e hepatotoxicidade da hipericina, um dos principais constituintes do Hypericum perforatum, comatividade antidepressiva (De Smet, 2002). Com relação ao Piper methysticum (kava-kava), há relatos de que seu consumo excessivo resultou em desnutrição, perda de peso, graves disfunções hepáticas e renais (Escher et al., 2001; Turolla \& Nascimento, 2006).

O uso de medicamentos na gestação é um evento freqüente (Schüler-Faccini et al., 2002). A resposta fetal, diante da medicação, é diferente da observada na mãe, podendo resultar em toxicidade, comlesões de variada monta, algumas irreversíveis (Gomes et al., 1999).

Reafirma-se, então, a importância dos estudos de significados e a possibilidade de contribuições das pesquisas de representação social ao campo da etnofarmacologia, comatenção, neste projeto, às representações formadas por gestantes.

\section{Representações das gestantes que usavam e das que não usavam plantas com finalidade medicinal na gravidez}

Nesse segmento, encontram-se os dados resultantes da análise de conteúdo feita na perspectiva bardiniana, apresentando-se, então, de acordo com essa perspectiva metodológica, as idéias e categorias identificadas, seguidas de falas das gestantes, que as exemplificame ilustram (Bardin, 1986).

Observaram-se, então, nos dados informados pelas gestantes, as dimensões da representação social, com atenção à atitude dos sujeitos, ou seja, ao julgamento de valor do objeto da representação (o uso medicinal de plantas), à informação, ou seja, o conhecimento a respeito do objeto, e o campo de representação, verificando-se, nessa dimensão, o seu núcleo, constituído das idéias mais constantes e comuns, que permitiram observar a ênfase no entendimento do significado das plantas medicinais e, nesse entendimento, os núcleos de sentido. Pôde-se, desse modo, identificar a categoria da concepção das plantas, associada à dos motivos do seu uso.

\section{usuárias \\ Representações do grupo de gestantes}

A dimensão de atitude, ou julgamento de valor do sujeito sobre o objeto da representação, foi predominantemente positiva nesse grupo de gestantes usuárias das plantas:
"Se da natureza tiramos as coisas necessárias à nossa vida, por que não os remédios?"

"...é natural, prefiro usar chá que remédio de farmácia."

"...eu me sentia melhorquando tomava o meu chazinho."

Quanto à dimensão de informação, verificase que a categoria concepção das plantas inclui a dos motivos de seu uso, como: remédio natural, tradicional (caseiro), eficaz, saudável, gratuito, confiável, sem química, "vem de Deus", simples e fácil de usar:

“... o remédio das plantas é válido pelo uso antigo da minha mãe..."

"...por hábito, desde criança, tomo todos os tipos de chá. Tudo que os outros 'fala' que 'era' bom, eu 'tô' bebendo."

É oportuno, também, lembrar que uma questão polêmica da sociologia é a que trata da classificação dicotômica das origens, naturais e sobrenaturais, das doenças e da cura (Paden, 2001). Pôde-se observar esse entendimento místico, ou sobrenatural, nas falas das gestantes, conforme se exemplificam a seguir:

"Acho muito bom, porque Deus colocou esses recursos na terra. E porque se eu for comprar todos os remédios, nem sempre tenho dinheiro."

"Eu sempre acreditei no poder de cura das plantas. Minha mãe, a vida toda dava pra gente tomar chá, xarope caseiro, essas coisas..."

"As plantas vieram de Deus, onosso criador."

Quanto ao possível uso de substâncias tóxicas, é importante lembrar que os produtos da biotransformação de vegetais têm elementos com potencial de toxicidade. O conhecimento etnofarmacológico aponta, por exemplo, que quanto mais "amarga" for a planta, maior o risco para o concepto. Contudo, apesar das ressalvas científicas, alguns vegetais revestidos de embriofetotoxicidade são utilizados pelas gestantes (Newall et al., 2002; Tan, 2008). Algumas falas significativas foram:

“...eu não estava preparada para engravidar, meu marido estava desempregado... foi uma senhora que me deu o chá amargo; já veio pronto, mas não me lembro o nome...

“...tomei melão-de-são-caetano, capim-limão e picão, porque o que amarga é bom pra nós e pra criança..."

"...era pra fazer uma mistura de erva bem amarga, mas não consegui tomar três vezes seguidas... tinha que tomar em jejum, mas 
não tomei. Minha avó que me deu. Era horrível..."

Ainda, na dimensão de informação, pôde-se identificar, nas categorias de concepção e motivos de uso, que as principais classes terapêuticas foram: analgésicos, antiespasmódicos, antiácidos, tranqüilizantes, afecções digestivas e respiratórias (antitussígenos/expectorantes). A maioria das gestantes relatou, também, o uso, com essas finalidades, de plantas sob a forma de chá, pela simplicidade do seu preparo.

A principal fonte do conhecimento sobre o uso medicinal de plantas foi a família, ao passo que apenas uma gestante $(0,7 \%)$ fazia uso de fitofármacos por orientação médica; $34 \%$ das gestantes usuárias de plantas durante a gravidez revelaram insegurança quanto ao uso correto da espécie vegetal; $77 \%$ desejavam aprender a utilizar melhor as plantas como medicamento e $76,3 \%$ gostariam de que os médicos receitassem medicamentos naturais.

$\mathrm{Na}$ dimensão do campo de representação, com atenção ao núcleo central, a identificação das idéias mais constantes e comuns, que constituem o núcleo, confirmam as análises feitas nas dimensões de atitude e informação. Pôde-se, desse modo, reafirmar, no campo de representação, as duas categorias, associadas e mutuamente recorrentes, da concepção das plantas e dos motivos do seu uso, evidenciando-se a ênfase na sua compreensão como envas medicinais, saudáveis, gratuitas, provenientes da natureza, de preparo caseiro, com resultados práticos eficazes, consagrados pela tradição familiar. As opiniões que se seguem são especialmente significativas, no sentido de ilustrar a análise:

"...porque eu acho melhor, tenho em casa, é barato e não faz tão mal."

"...como é planta, acho que não faz mal à criança e faz bem à saúde."

"...uso para não ter que usar remédio de farmácia, porque esses prejudicam e você não pode usar se não for comreceita médica." "Eu tive muito enjôo no começo da gravidez e tomei chá de boldo, porque é muito mais barato que remédio...".

"Tenho muito mais confiança na planta do que em remédio de farmácia".

Essas afirmações exemplificam, demonstram e confirmam as idéias que se associam nas categorias de concepção e motivos: uso natural, caseiro, eficaz, saudável, gratuito, confiável.

Com essas idéias comuns nas representações do grupo de gestantes que usavam plantas medicinais, chega-se, então, à análise dos temas que se apresentaram nas representações do grupo que não fazia uso de plantas.

\section{Representações do grupo de gestantes não usuárias}

Destacaram-se também, nesse grupo, as categorias, associadas, da concepçãoe dos motivos, nucleados nas seguintes idéias: zelo pela gravidez saudável, desconhecimento da fitomedicina, atenção às recomendações obstétricas e avaliação das plantas medicinais como perigosas ou tóxicas, podendo prejudicar o feto:

"Tenho medo de abortar porque não se sabe o que a planta pode fazer".

"Eu fazia pré-natal e quando tinha alguma coisa o médico passava remédio, mesmo".

“Não teve 'precisão' de usar, eu não senti nada... minhas 'gravidez'foram ótimas..."

"Não conheço bem as ervas e tenho medo...elas podem fazer mal."

As idéias que se apresentaramna categoria dos motivos que justificam não usar as plantas demonstram, também, a importância que as gestantes atribuem às orientações do médico e a preocupação de usarem plantas sem a sua recomendação:

"Não vou usar plantas, pois a doutora não passou e elas podem fazer mal".

"Senti muita cólica e enjôo, mas só tomei os remédios receitados pela médica."

"Não tomei, não, primeiro porque eu tenho medo de dar algum problema e segundo porque eu tenho problema de pressão alta e o médico cortou tudo..."

Comesses dados da representação do uso medicinal de plantas, pelos dois grupos de gestantes, podem-se formular as considerações finais da pesquisa, nas quais se reafirmam ou se complementam os elementos de análise e de recomendações que o estudo sugere aos profissionais da saúde.

\section{CONSIDERACCÕES FINAIS}

Este estudo evidenciou que o uso de plantas, com finalidade medicinal, na gravidez, pode ser analisado a partir da compreensão de representações das usuárias, que informam sobre fatores que influenciam esse uso, assimcomo das não usuárias, que informam os motivos pelos quais não as utilizam. A informação é expressa em linguagem de senso comum, usada nas comunicações e interações cotidianas, enquanto processos através dos quais formam-se e consolidam-se as representações (Moscovici, 1978; Jodelet, 2001; Guareschi, 1995).

Assim, as análises das representações do grupo de gestantes usuárias permitiram observar 
hábitos peculiares, resultantes da influência da tradição familiar, no sentido do uso de chás, preparados com várias espécies vegetais, consideradas, concomitantemente, úteis e "inofensivas" na gravidez.

No percurso da análise das dimensões das representações e das unidades de sentido identificadas e exemplificadas nas falas das gestantes, configurando as categorias de sua concepção e seus motivos, observou-se que o grupo de usuárias de plantas enfatizou o seu entendimento como ervas medicinais, de qualidade "natural", com ação ou resultados práticos efetivos, comprovados pela tradição do seu uso pela família, como coloca Medeiros \& Cabral, 2001.

Para esse grupo, o medicamento parece possuir, não só uma função farmacológica, mas também cumprir um papel simbólico, de garantir às gestantes os efeitos positivos da ação terapêutica.

Quanto ao grupo de gestantes que não utilizavam fitofármacos, constatou-se, especialmente, nas suas representações, que sua concepção e motivos expressavamum sentimento de desconfiança emplantas para uso medicinal, coerente coma idéia de que somente são eficazes os medicamentos prescritos pelos médicos obstetras. Esse dado também encontra-se em estudos como o de Osóriode-Castro et al. (2004), valendo tambémobservar, em pesquisa francesa, que $99 \%$ das mulheres pesquisadas receberam prescrição de, pelo menos, um medicamento durante a gestação (Lacroix et al., 2000). No Brasil, pesquisa de Gomes et al. (2000) apontou a média de 4,2 medicamentos por gestante.

É também oportuno observar, nessas considerações finais, que nem sempre as plantas vendidas com um nome popular representam a mesma espécie botânica e, ainda que conhecidos, popularmente, pela mesma denominação, vegetais semelhantes podem não possuir as mesmas propriedades, sendo possível, inclusive, que possuam potenciais adaptógenos (Mendes \& Carlini, 2007), ou contenham um considerável teor de toxicidade (Belew, 1999).

Finalmente, assinala-se que cabe aos profissionais de saúde informar às mulheres emidade fértil sobre o risco, assim como sobre possibilidades da utilização de plantas como medicamentos na gravidez, chamando, sobretudo, a atenção para o risco potencial da automedicação (Barns, 2003).

Reafirma-se, então, a importância do diálogo e da orientação dos médicos, associada ao estudo científico das plantas medicinais. Assim, conhecendose o perfil dos medicamentos usados na gravidez, podem-se planejar intervenções educativas dirigidas a gestantes, assim como atividades de educação continuada para profissionais de saúde (Stuttard, 2002).

\section{REFERÊNCIA}

ALBUQUERQUE, U.P.; HANAZAKI, N. As pesquisas etnodirigidas na descoberta de novos fármacos de interesse médico e farmacêutico: fragilidades e pespectivas. Revista Brasileira de Farmacognosia, v.16, supl., p.678-89, 2006.

ANDRADE, S.E. et al. Prescription drug use in pregnancy. American Journal of Obstetrics and Gynecology,v. 191, n.2, p.398-407, 2004.

ARRUDA, A. Teoria das representações sociais e teorias de gênero. Cadernos de Pesquisa, v.117, p.127-47, 2002. ASCHWANDEN, C. Herbs for health, but how safe are they? Bulletin of the World Health Organization, v.79, n.7, p.691-2, 2001. Disponível em: <http://www. who.int/ bulletin>. Acesso em: 08 mar. 2008.

BAIÃO, M.R.; DESLANDESS.F. Alimentação na gestação e puerpério. Revista de Nutrição, v.19, p.245-53, 2007. BARDIN, L. Análise de conteúdo. Lisboa: Edições 70, 1986. 233p.

BARNS, J. Complementary therapies in pregnancy. The Pharmaceutical Journal, v.270, p.402-4, 2003.

BELEW, C. Herbs and the childbearing woman guidelines for midwives. Journal of Nurse-Midwifery, v.44, n.3, p.231-52, 1999.

BRASIL. Ministério da Saúde. Agência Nacional de Vigilância Sanitária. Resolução RDC 48, de 16 de março de 2004. Dispõe sobre o registro de medicamentos fitoterápicos. Diário Oficial da União, Brasília, 18 mar. 2004. Disponível em <http://e-legis.anvisa.gov.br/leisref/ public/showAct. php?id=10230\&word $>$. Acesso em: 08 mar. 2008.

BRASIL. Ministério da Saúde. Secretaria de Ciência, Tecnologia e Insumos Estratégicos. Departamento de Assistência Farmacêutica. Política nacional de plantas medicinais e fitoterápicos. Brasília: Ministério da Saúde, 2006. 60p.

BUGNO, A. et al. Evaluation of microbiological contamination of crude herbal drugs. Revista Brasileira de Ciências Farmacêuticas, v.41, p.491-7, 2005.

CALVASINA, P.G. et al. "Fraqueza de nascença": sentidos e significados culturais de impressões maternas na saúde infantil no Nordeste brasileiro. Cadernos de Saúde Pública, v.23, p.371-80, 2007.

CARVALHO, A.C.B. et al. Aspectos da legislação no controle dos medicamentos fitoterápicos. T\&C Amazônia, v.5, n.11, p.26-32, 2007.

CASTRO, H.G.; FERREIRA, F.A. A dialética do conhecimento no uso das plantas medicinais. Revista Brasileira de Plantas Medicinais, v.2, p.19-21, 2001.

CECATTI, J.C. Saúde da mulher: enfoque da evidência científica para a prevenção da morbidade e mortalidade materna. Revista Brasileira de Saúde Materno-Infantil, v.5, n.1, p.9-11, 2005.

CHOUDHURI, S.; VALERIO, L.G.J. Usefulness of studies on the molecular mechanism of action of herbals/ botanicals: the case of St. John's wort. Journal of Biochemical and Molecular Toxicology, v.19, n.1, p.1$11,2005$.

DAVID, J.P.L.; DAVID, J.M. Plantas medicinais: fármacos derivados de plantas. In: SILVA, P. (Org.). Farmacologia. São Paulo: Guanabara Koogan, 2006. p.134-45. DE SMET, P.A.G.M. Herbal remedies. The New England 
Journal of Medicine, v.347, n.25, 2046-56, 2002.

DI STASI, L.C. Medicinal plants popularly used in the Brazilian Tropical Atlantic Forest. Fitoterapia, v.73, p.6991, 2002.

DUTAU, G. Le risque allergique des produits diététiques et médicinaux. Revue Française d'Allergologie et d'Immunologie Clinique, v.44, p.634-45, 2004.

ERNST, E. The risk-benefit profile of commonly used herbal therapies: ginkgo, st. john's wort, ginseng, echinacea, saw palmetto, and kava. Annals of Internal Medicine, v.136, n.1, p.42-53, 2002.

ESCHER, M. et al. Hepatitis associated with Kava, a herbal remedy for anxiety. British Medical Journal, v.322, p.139, 2001.

ETKIN, N.L.; ELISABETSKY, E. Seeking a transdisciplinary and culturally germane science: the future of ethnopharmacology. Journal of Ethnopharmacology, v.100,n.2, p.23-6, 2005.

FONSECA, M.; FONSECA, E.;BERGSTEN-MENDES, G. Prevalência do uso de medicamentos na gravidez: uma abordagem farmacoepidemiológica. Revista de Saúde Pública, v.36, n.2, p.205-12, 2002.

FUGH-BERMAN, A. Herb-drug interactions. Lancet, v.355, p.134-8, 2000.

FUGH-BERMAN, A.; LIONE, A.; SCIALLI,A.R. Do no harm: avoidance of herbal medicines during pregnancy. Obstetrics \& Gynecology, v.105, p.1119-22, 2005.

GIBSON P.S. Herbal and alternative medicine use during pregnancy: a cross-sectional survey. Obstetrics \& Gynecology, v.97, n.4, supl., p.41-2, 2001.

GOFFMAN, E. A representação na vida cotidiana.3.ed. Petrópolis: Vozes, 2002. 233p.

GOMES K.R.O. et al. Prevalência do uso de medicamentos na gravidez e relações com as características maternas. Revista de Saúde Pública, v.33, n.3, p.246-54, 2000

GUARESCHI, P. (Coord). Comunicação e controle social. 3.ed. Petrópolis: Vozes. 2000.71p.

GUARESCHI, P. "Sem dinheiro não há salvação": ancorando o bem e o mal entre os neopentecostais. In: JOVCHELOVITCH, S.; GUARESCHI, P. (Orgs.). Textos em representações sociais. Petrópolis: Vozes, 1995. p.191-228.

GUERRA, M.P.; NODARI, R.O. Biodiversidade: aspectos biológicos, geográficos, legais e éticos. In: SIMÕES, C.M.O. (Org.). Farmacognosia: da planta ao medicamento. Porto Alegre: UFRGS/UFSC, p.13-26, 2001.

JODELET, D. (Org.). As representações sociais: um domínio em expansão. Rio de Janeiro: EDUERJ, 2001. 420p.

LACROIX, I. et al. Prescription of drugs during pregnancy in France. Lancet, v.356, p.1735-6, 2000.

LALOO, R.C. et al. Status of medicinal plants in the disturbed and the undisturbed sacred forests of Meghalaya, northeast India: population structure and regeneration efficacy of some important species. Current Science, v.90, p.225-32, 2006.

MENDONÇA FILHO, R.F.W.; MENEZES, F.S. Estudo da utilização de plantas medicinais pela população da Ilha Grande (RJ). Revista Brasileira de Farmacognosia, v.13, supl., p.55-8, 2003.

MEDEIROS, L.C.M.; CABRAL, I.E. O cuidar com plantas medicinais: uma modalidade de atenção à criança pelas mães e enfermeira-educadora. Revista LatinoAmericana de Enfermagem, v.9, n.1, p.18-26, 2001. MENDES, F.R., CARLINI, E.A. Brazilian plants as possible adaptogens: an ethnopharmacological survey of books edited in Brazil. Journal of Ethnopharmacology, v.109, p.493-500, 2007.

MINAYO, M.C.S. O desafio do conhecimento:pesquisa qualitativa em saúde. São Paulo: Hucitec-ABRASCO, 2006. 406p.

MOSCOVICI, S. A representação social da psicanálise. Rio de Janeiro: Zahar, 1978. 391p.

MOSCOVICI, S.. Notes towards a description of social representations. European Journal of Social Psychology, v.18, p.11-250, 1988.

MOSCOVICI, S. Das representações coletivas às representações sociais: elementos para uma história. In: JODELET, D. (Org.). As representações sociais. Rio de Janeiro: EDUERJ, 2001. p.45-66.

MOSCOVICI, S. Representações sociais - investigação em psicologia social. Petrópolis: Vozes, 2005. 408p.

NEWALL C.A.; ANDERSON, L.A.; PHILLIPSON, J.D. Plantas Medicinais: guia para profissional de saúde. São Paulo: Premier, 2002. 308p.

ORGANIZAÇÃO PANAMERICANA DE SAÚDE. World Health Organization, Division of Health Systems and Services Development. Traditional, complementary and alternative medicine and therapies in the Americas: policies, plans and programs. Washington, D.C.: PAHO, 2002. 26p.

ORGANIZAÇÃO PANAMERICANADE SAÚDE. Plantio, coleta e manuseio de plantas medicinais. Agenda OPAS-OMS, mar./abr. 2004. Disponível em: <http:// www.opas.org.br/sistema/arquivos/agenda11.pdf $>$. Acesso em: 26 fev. 2008.

OSORIO-DE-CASTRO, C.G.S.;PAUMGARTTEN, F.J.R.; SILVER, L.D. O uso de medicamentos na gravidez. Ciência \& Saúde Coletiva, v.9, n.4, p.987-96, 2004.

PADEN, W.E. Interpretando o sagrado: modos de conceber a religião. São Paulo: Paulinas, 2001. 240p. PERINI, E.; MAGALHÃES, S.M.S.; NORONHA, V. Consumo de medicamentos no período de internação para o parto. Revista de Saúde Pública, v.39, n.3, p.358-65, 2005.

PINN, G.; PALLETT, L. Herbal medicine in pregnancy. Complementary Therapies in Nursing \& Midwifery, v.8, p.77-80, 2002.

RANGEL, M. A pesquisa de representação social no enfrentamento de problemas socioeducacionais. Aparecida: Idéias \& Letras, 2004. 72p.

SÁ, C. A construção do objeto de pesquisa em representações sociais. Rio de Janeiro: EDUERJ, 1998. $110 p$.

SÁ, C. Núcleo central das representações sociais. Petrópolis: Vozes, 1996. 189p.

SANTIAGO, R. A escola representada pelos alunos, pais e professores. Aveiro: Universidade de Aveiro, 2000. $189 p$.

SCHOPENHAUER, A. O mundo com vontade e representação. Rio de Janeiro: Contraponto, 2001. 432p.

SCHÜLER-FACCINI, L. et al. Avaaliação de teratógenos na população brasileira. Ciência \& Saúde Coletiva, v.7, n.1, p.65-71, 2002 
SOUZA, L.F. Recursos vegetais usados na medicina tradicional do Cerrado (comunidade de Baús, Acorizal, MT, Brasil). Revista Brasileira de Plantas Medicinais, v.9, n.4, p.44-54, 2007

SPINK, M.J. Desvendando as teorias implícitas: uma metodologia de análise das representações sociais. In: JOVCHELOVITCH, S.;GUARESCHI, P. (Orgs.). Textos em representações sociais. Petrópolis: Vozes, 1995. p.117-48.

STICKEL, F.; SCHUPPAN, D. Herbal medicine in the treatment of liver diseases. Digestive and Liver Disease, v.39, p.293-304, 2007.

STUTTARD, P. Education and training of complementary therapists: the role of higher education. Complementary Therapies in Nursing \& Midwifery, v.8, p.106-12, 2002.

TAN, P. et al. Teratogenic effects, acute and sub chronic toxicity of the leaf aqueous extract of Ocimum suave Wild (Lamiaceae) in rats. Journalof Ethnopharmacology, v.115, p.232-7, 2008.

TESKE, M.; TRENTINI, A.M. Herbarium: compêndio de fitoterapia. Curitiba: Herbarium Laboratório Botânico, 2001.317p.

TIRAN, D. The use of herbs by pregnant and childbearing women: a risk-benefit assessment. Complementary Therapies in Nursing \& Midwifery, v.9, p.176-81, 2003. TUROLLA, M.S.R.; NASCIMENTO, E.S. Informações toxicológicas de alguns fitoterápicos utilizados no Brasil. Revista Brasileira de Ciências Farmacêuticas, v.42, n.2, p.289-306, 2006.

VALA, J. Sobre as representações sociais: para uma epistemologia do senso comum. Lisboa: Universidade de Lisboa, p.5-28, 1986. (Cadernos de Ciências Sociais). 